\title{
CR- Submanifoldsof a Nearly Hyperbolic Cosymplectic Manifold
}

\author{
Mobin Ahmad and Kashif Ali \\ Department of Mathematics, Integral University, Kursi Road, Lucknow-226026, India.
}

\begin{abstract}
In the present paper, we study some properties of CR-submanifolds of a nearly hyperbolic cosymplectic manifold. We also obtain some results on $\xi$-horizontal and $\xi-v e r t i c a l$ CR-submanifolds of a nearly hyperboliccosymplectic manifold.
\end{abstract}

Keywords: CR-submanifolds, nearlyhyperbolic cosymplectic manifold, totally geodesic,parallel distribution.

\section{Introduction}

The notion of CR-submanifolds of Kaehler manifold was introduced and studied by A. Bejancu in ([1], [2]). Since then, several papers on Kaehler manifolds were published. CR-submanifolds of Sasakian manifold was studied by C.J. Hsu in [3] and M. Kobayashi in [4].Later, several geometers (see, [5], [6] [7], [8] [9], [10]) enriched the study of CR-submanifolds of almost contact manifolds. On the other hand,almost hyperbolic $(f, g, \eta, \xi)$-structure was defined and studied by Upadhyay and Dube in [11]. Dube and Bhatt studied CRsubmanifolds of trans-hyperbolic Sasakian manifold in [12]. In this paper, we study some properties of CRsubmanifolds of a nearly hyperbolic cosymplectic manifold.

The paper is organized as follows. In section 2, we give a brief description of nearly hyperbolic cosymplectic manifold.In section 3, some properties of CR-submanifolds of nearly hyperbolic cosymplectic manifold are investigated. In section 4 , some results on parallel distribution on $\xi$-horizontal and $\xi$-vertical CR- submanifolds of a nearly cosymplectic manifold are obtained.

\section{Nearly Hyperbolic Cosymplectic manifold}

Let $\bar{M}$ be an $n$-dimensional almost hyperbolic contact metric manifold with the almost hyperbolic contact metric $(\phi, \xi, \eta, g)$ - structure, where a tensor $\phi$ of type $(1,1)$ a vector field $\xi$, called structure vector field and $\eta$, the dual 1-form of $\xi$ satisfying the followings:

$$
\begin{aligned}
& \phi^{2} X=X+\eta(X) \xi, \quad g(X, \xi)=\eta(X),(2.1) \\
& \eta(\xi)=-1, \quad \phi(\xi)=0, \quad \eta o \phi=0,(2.2) \\
& g(\phi X, \phi Y)=-g(X, Y)-\eta(X) \eta(Y)(2.3)
\end{aligned}
$$

for any $X, Y$ tangent to $\bar{M}[11]$. In this case

$$
g(\phi X, Y)=-g(X, \phi Y) \cdot(2.4)
$$

An almost hyperbolic contact metric $(\phi, \xi, \eta, g)$-structure on $\bar{M}$ is called nearly hyperbolic cosymplecticstructure if and only if

$$
\left(\bar{\nabla}_{X} \phi\right) Y+\left(\bar{\nabla}_{Y} \phi\right) X=0,(2.5)
$$

$\bar{\nabla}_{X} \xi=0(2.6)$

for all $X, Y$ tangent to $\bar{M}$ and Riemannian Connection $\bar{\nabla}$.

\section{CR-Submanifolds of Nearly Hyperbolic Cosymplectic Manifold}

Let $M$ be a submanifold immersed in $\bar{M}$. We assume that the vector field $\xi$ is tangent to $M$. Then $M$ is called a CR-submanifold [13] of $\bar{M}$ if there exist two orthogonal differentiable distributions $D$ and $D^{\perp}$ on $M$ satisfying

(i) $T M=D \oplus D^{\perp}$,

(ii) the distribution $D$ is invariant by $\phi$, that is, $\phi D_{X}=D_{X}$ for each $X \epsilon M$,

(iii) the distribution $D^{\perp}$ is anti-invariant by $\phi$, that is, $\phi D_{X}^{\perp} \subset T_{X} M^{\perp}$ for each $X \epsilon M$,

whereTMand $T^{\perp} M$ be the Lie algebra of vector fields tangential to $M$ and normal to $M$ respectively. If $\operatorname{dim} D_{x}^{\perp}=0\left(\right.$ resp., $\left.\operatorname{dim} D_{x}=0\right)$, then the CR-submanifold is called an invariant (resp., anti-invariant) submanifold. The distribution $D\left(\right.$ resp.,$\left.D^{\perp}\right)$ is called the horizontal (resp., vertical)distribution. Also, the pair $\left(D, D^{\perp}\right)$ is called $\xi$ - horizontal(resp., vertical)if $\xi_{X} \in D_{X}\left(\right.$ resp.,$\left.\xi_{X} \in D_{X}^{\perp}\right)$.

Let the Riemannian metric induced on $M$ is denoted by the same symbol $g$ and $\nabla$ be the induced LeviCivita connection on $N$, then the Gauss and Weingarten formulas are given respectively by [14]

$$
\begin{aligned}
& \bar{\nabla}_{X} Y=\nabla_{X} Y+h(X, Y),(3.1) \\
& \bar{\nabla}_{X} N=-A_{N} X+\nabla_{X}^{1} N(3.2)
\end{aligned}
$$


for any $X, Y \in T M a n d N \in T^{\perp} M$, where $\nabla^{\perp}$ is a connection on the normal bundle $T^{\perp} M, h$ is the second fundamental form and $A_{N}$ is the Weingarten map associated with $\mathrm{N}$ as $g\left(A_{N} X, Y\right)=g(h(X, Y), N)(3.3)$

for any $x \in \operatorname{Mand} X \in T_{x} M$. We write $X=P X+Q X,(3.4)$

where $P X \in D$ Dand $Q X \in D^{\perp}$.

Similarly, for $N$ normal to $M$, we have $\phi N=B N+C N,(3.5)$

whereBN(resp. $C N$ ) is the tangential component (resp.normalcomponent) of $\phi N$.

Lemma 3.1.Let $M$ be a CR- submanifold of a nearly hyperbolic cosymplectic manifold $\bar{M}$.Then $\phi P\left(\nabla_{X} Y\right)+\phi P\left(\nabla_{Y} X\right)=P \nabla_{X}(\phi P Y)+P \nabla_{Y}(\phi P X)-P A_{\phi Q Y} X-P A_{\phi Q X} Y,(3.6)$

$2 B h(X, Y)=Q \nabla_{X}(\phi P Y)+Q \nabla_{Y}(\phi P X)-Q A_{\phi Q X} Y-Q A_{\phi Q Y} X,(3.7)$

$\phi Q \nabla_{X} Y+\phi Q \nabla_{Y} X+2 C h(X, Y)=h(X, \phi P Y)+h(Y, \phi P X)+\nabla_{X}^{\frac{1}{X}} \phi Q Y+\nabla_{Y}^{\frac{1}{Y}} \phi Q X(3.8)$

forany $X, Y \in T M$.

Proof.Using (2.4), (2.5) and (2.6), we get $\left(\bar{\nabla}_{X} \phi\right) Y+\phi\left(\nabla_{X} Y\right)+\phi h(X, Y)=\nabla_{X}(\phi P Y)+h(X, \phi P Y)-A_{\phi Q Y} X+\nabla_{X}^{\frac{1}{X}} \phi Q Y$.

Interchanging $X \& Y$ and adding, we have

$$
\begin{aligned}
\left(\bar{\nabla}_{X} \phi\right) Y+\left(\bar{\nabla}_{Y} \phi\right) X & +\phi\left(\nabla_{X} Y\right)+\phi\left(\nabla_{Y} X\right)+2 \phi h(X, Y) \\
& =\nabla_{X}(\phi P Y)+\nabla_{Y}(\phi P X)+h(X, \phi P Y)+h(Y, \phi P X)
\end{aligned}
$$

$-A_{\phi Q Y} X-A_{\phi Q X} Y+\nabla_{X}^{\frac{1}{X}} \phi Q Y+\nabla_{Y}^{\frac{1}{Y}} \phi Q X$.

Using (2.5) in above equation, we have

$$
\begin{gathered}
\phi P\left(\nabla_{X} Y\right)+\phi Q\left(\nabla_{X} Y\right)+\phi P\left(\nabla_{Y} X\right)+\phi Q\left(\nabla_{Y} X\right)+2 B h(X, Y) \\
+2 C h(X, Y)=P \nabla_{X}(\phi P Y)+Q \nabla_{Y}(\phi P X)+h(X, \phi P Y) \\
+h(Y, \phi P X)-P A_{\phi Q Y} X-Q A_{\phi Q Y} X-P A_{\phi Q X} Y \\
-Q A_{\phi Q X} Y+\nabla_{X}^{\perp} \phi Q Y+\nabla_{Y}^{\perp} \phi Q X .(3.9)
\end{gathered}
$$

Comparing the horizontal, vertical and normal components, we get (3.6) - (3.8).

Hence the Lemma is proved.

Lemma 3.2.Let $M$ be a CR- submanifold of a nearly hyperbolic cosymplectic manifold $\bar{M}$. Then $2\left(\bar{\nabla}_{X} \phi\right) Y=\nabla_{X} \phi Y-\bar{\nabla}_{Y} \phi X+h(X, \phi Y)-\nabla_{Y} \phi X-\phi[X, Y](3.10)$

Proof.From Gauss formula (3.1), we have forany $X, Y \in D$.

Also, we have

$$
\bar{\nabla}_{X} \phi Y-\bar{\nabla}_{Y} \phi X=\nabla_{X} \phi Y+h(X, \phi Y)-\nabla_{Y} \phi X-h(Y, \phi X) .(3.11)
$$

$$
\bar{\nabla}_{X} \phi Y-\bar{\nabla}_{Y} \phi X=\left(\bar{\nabla}_{X} \phi\right) Y-\left(\bar{\nabla}_{Y} \phi\right) X+\phi[X, Y] \text {. (3.12) }
$$

From (3.11) and (3.12), we get

$$
\left(\bar{\nabla}_{X} \phi\right) Y-\left(\bar{\nabla}_{Y} \phi\right) X=\nabla_{X} \phi Y+h(X, \phi Y)-\nabla_{Y} \phi X-h(Y, \phi X)-\phi[X, Y] .
$$

Adding (3.15) and (2.5), we obtain

Hence the Lemma is proved.

$$
2\left(\bar{\nabla}_{X} \phi\right) Y=\nabla_{X} \phi Y+h(X, \phi Y)-\nabla_{Y} \phi X-h(Y, \phi X)-\phi[X, Y] .
$$

Lemma 3.3.Let $M$ be a CR- submanifold of a nearly hyperbolic cosymplectic manifold $\bar{M}$. Then

$$
\begin{gathered}
2\left(\bar{\nabla}_{X} \phi\right) Y=A_{\phi X} Y-A_{\phi Y} X+\nabla_{X}^{1} \phi Y-\nabla_{Y}^{\perp} \phi X-\phi[X, Y](3.14) \\
\text { forany } X, Y \in D^{\perp} .
\end{gathered}
$$

Proof.From Weingarten formula (3.2), we have

$$
\bar{\nabla}_{X} \phi Y-\bar{\nabla}_{Y} \phi X=A_{\phi X} Y-A_{\phi Y} X+\nabla_{X}^{\frac{1}{X}} \phi Y-\nabla_{Y}^{\frac{1}{}} \phi X .(3.15)
$$

Also,

$$
\bar{\nabla}_{X} \phi Y-\bar{\nabla}_{Y} \phi X=\left(\bar{\nabla}_{X} \phi\right) Y-\left(\bar{\nabla}_{Y} \phi\right) X+\phi[X, Y] .(3.16)
$$

From (3.15) and (3.16), we get

$$
\left(\bar{\nabla}_{X} \phi\right) Y-\left(\bar{\nabla}_{Y} \phi\right) X=A_{\phi X} Y-A_{\phi Y} X+\nabla_{X}^{\frac{1}{}} \phi Y-\nabla_{Y}^{\perp} \phi X-\phi[X, Y] \text {. (3.17) }
$$

Adding (3.17) and (2.5), we obtain

$$
2\left(\bar{\nabla}_{X} \phi\right) Y=A_{\phi X} Y-A_{\phi Y} X+\nabla_{X}^{\frac{1}{X}} \phi Y-\nabla_{Y}^{\frac{1}{}} \phi X-\phi[X, Y] .
$$

Hence the Lemma is proved.

Lemma 3.4.Let $M$ be a CR- submanifold of a nearly hyperbolic cosymplectic manifold $\bar{M}$. Then $2\left(\bar{\nabla}_{X} \phi\right) Y=-A_{\phi Y} X+\nabla_{X}^{\frac{1}{X}} \phi Y-\nabla_{Y} \phi X-h(Y, \phi X)-\phi[X, Y]$ (3.18)

forany $X \in \operatorname{Dand} Y \in D^{\perp}$. 
Proof.Using Gauss and Weingarten formula for $\in \operatorname{Dand} Y \in D^{\perp}$, we have

Also, we have

$$
\bar{\nabla}_{X} \phi Y-\bar{\nabla}_{Y} \phi X=-A_{\phi Y} X+\nabla_{X}^{\frac{1}{X}} \phi Y-\nabla_{Y} \phi X+h(Y, \phi X) \text {. (3.19) }
$$

$$
\bar{\nabla}_{X} \phi Y-\bar{\nabla}_{Y} \phi X=\left(\bar{\nabla}_{X} \phi\right) Y-\left(\bar{\nabla}_{Y} \phi\right) X+\phi[X, Y] .(3.20)
$$

By virtue of (3.19) and (3.20), we get

$$
\left(\bar{\nabla}_{X} \phi\right) Y-\left(\bar{\nabla}_{Y} \phi\right) X=-A_{\phi Y} X+\nabla_{X}^{1} \phi Y-\nabla_{Y} \phi X+h(Y, \phi X)-\phi[X, Y] .(3.21)
$$

Adding (3.21) and (2.5), we obtain

$$
2\left(\bar{\nabla}_{X} \phi\right) Y=-A_{\phi Y} X+\nabla_{X}^{1} \phi Y-\nabla_{Y} \phi X+h(Y, \phi X)-\phi[X, Y] .
$$

Hence the Lemma is proved.

\section{Parallel Distribution}

Definition4.1.The horizontal (resp., vertical) distribution $D\left(\right.$ resp.,$\left.D^{\perp}\right)$ is said to be parallel [13] with respect to the connectionon $\operatorname{Mif}_{X} Y \in D\left(\right.$ resp., $\left.\nabla_{Z} W \in D^{\perp}\right)$ for any vector field $X, Y \in D\left(\right.$ resp., $\left.W, Z \in D^{\perp}\right)$.

Theorem 4.2. Let $M$ be a $\xi$-vertical CR-submanifold of a nearly hyperbolic cosymplecticmanifold $\bar{M}$. If the horizontal distribution $D$ is parallel,then

$$
h(X, \phi Y)=h(Y, \phi X) .(4.1)
$$

forany $X, Y \in D$.

Proof.Using parallelism of horizontal distribution $\mathrm{D}$, we have

$$
\nabla_{X}(\phi Y) \in \operatorname{Dand}_{Y} \phi X \in D \text { forany } X, Y \in D .(4.2)
$$

Now, by virtue of (3.7), we have

$$
B h(X, Y)=0 .(4.3)
$$

From (3.5) and (4.3), we get

forany $X, Y \in D$.

$$
\phi h(X, Y)=\operatorname{Ch}(X, Y)(4.4)
$$

From (3.8), we have

$$
h(X, \phi Y)+h(Y, \phi X)=2 C h(X, Y)(4.5)
$$

forany $X, Y \in D$.

Replacing $X b y \phi X i n$ (4.5) and using (4.4), we have $h(\phi X, \phi Y)+h(Y, X)=\phi h(\phi X, Y)$. (4.6)

Now, replacing $Y$ by $\phi Y$ in (4.6), we get

$$
h(X, Y)+h(\phi Y, \phi X)=\phi h(X, \phi Y) .(4.7)
$$

Thus from (4.6) and (4.7), we find

$$
h(X, \phi Y)=h(Y, \phi X) \text {. }
$$

Hence the Theorem is proved.

Theorem 4.3.Let $M$ be aCR-submanifold of a nearly hyperbolic cosymplecticmanifold $\bar{M}$. If the distribution $D^{\perp}$ is parallel with respect to the connection on $\mathrm{M}$, then

$$
\begin{aligned}
& A_{\phi Y} Z+A_{\phi Z} Y \in D^{\perp} \\
& \text { forany } Y, Z \in D^{\perp} .
\end{aligned}
$$

Proof.Let $Y, Z \in D^{\perp}$, then using (3.1) and (3.2), we have

$$
-A_{\phi Z} Y-A_{\phi Y} Z+\nabla_{Y}^{\frac{1}{Y}} \phi Z+\nabla_{Z}^{\frac{1}{Z}} \phi Y=\phi\left(\nabla_{Y} Z\right)+\phi \nabla_{Z} Y+2 \phi h(Y, Z) \text {.(4.8) }
$$

Taking inner product with $X \in \operatorname{Din}(4.8)$, we get

$$
g\left(A_{\phi Y} Z+A_{\phi Z} Y\right)=0
$$

which is equivalent to

$$
\left(A_{\phi Y} Z+A_{\phi Z} Y\right) \in D^{\perp}
$$

forany $Y, Z \in D^{\perp}$.

Definition 4.4.A CR-submanifold is said to be mixed-totally geodesic ifh $(X, Z)=0$ forall $X \in \operatorname{Dand} Z \in D^{\perp}$.

Lemma 4.5. Let $M$ be a CR-submanifold of a nearly hyperbolic cosymplecticmanifold $\bar{M}$. Then $M$ is mixed totally geodesic if and only if $A_{N} X \in D$ for all $X \in D$.

Definition 4.6.A Normal vector field $N \neq 0$ is called $D-$ parallel normal section if $\nabla_{X}^{\frac{1}{X}} N=0$ for all $X \in D$.

Theorem 4.7.Let $M$ be a mixed totally geodesic CR-submanifold of a nearly hyperbolic cosymplecticmanifold $\bar{M}$. Then the normal section $N \in \phi D^{\perp}$ is $D-$ parallel if and only if $\nabla_{X} \phi N \in D$ forallX $\in$ $D$.

Proof.Let $N \in \phi D^{\perp}$, then from (3.7), we have $Q \nabla_{Y} \phi X=0$.

In particular, we have $Q \nabla_{Y} \phi X=0$. Using it in (3.8), we have $\phi Q \nabla_{X} \phi N=\nabla_{X}^{\frac{1}{X}} N$. (4.9) 
Thus, if the normal section $N \neq 0$ is D-parallel, then using 'definition 4.6' and (4.9), we get

$$
\phi \nabla_{X}(\phi N)=0
$$

which is equivalent to $\nabla_{X}(\phi N) \in 0$ forall $X \in D$.

The converse part easily follows from (4.9). This completes the proof of the theorem.

\section{References}

[1]. A.Bejancu, CR-submanifolds of a Kaehler manifold I, Proc. Amer. Math. Soc. 69 (1978), 135-142.

[2]. CR-submanifolds of a Kaehler manifold II, Trans. Amer. Math. Soc. 250 (1979), 333-345.

[3]. C.J. Hsu, On CR-submanifolds of Sasakian manifolds I, Math. Research Center Reports, Symposium Summer 1983, 117-140.

[4]. M. Kobayash, CR-submanifolds of a Sasakian manifold, Tensor N.S. 35 (1981), 297-307.

[5]. A. Bejancuand N.Papaghuic, CR-submanifolds of Kenmotsu manifold, Rend. Mat.7 (1984), 607-622.

[6]. Lovejoy S.K. Das and M. Ahmad, CR-submanifolds of LP-Sasakian manifolds with quarter symmetric non-metric connection, Math. Sci. Res. J. 13 (7), 2009, 161-169.

[7]. C. Ozgur, M. Ahmad and A. Haseeb, CR-submanifolds of LP-Sasakian manifolds with semi-symmetric metric connection, Hacettepe J. Math. And Stat. vol. 39 (4) (2010), 489-496.

[8]. K. Matsumoto, On CR-submsnifolds of locally conformal Kaehler manifolds, J. Korean Math. Soc. 21 (1984), $49-61$.

[9]. M. Ahmad and J.P. Ojha, CR-submanifolds of LP-Sasakian manifolds with the canonical semi-symmetric semi-metric connection, Int. J. Contemp. Math. Science, vol.5 (2010), no. 33, 1637-1643.

[10]. M. Ahmad, M.D. Siddiqi and S. Rizvi, CR-submanifolds of a nearly hyperbolic Sasakian manifold admitting semi-symmetric semimetric connection, International J. Math. Sci. \&Engg.Appls., Vol. 6 (2012), 145-155.

[11]. M.D. Upadhyayand K.K. Dube, Almost contact hyperbolic $(\phi, \xi, \eta, g)$-structure, Acta. Math. Acad. Scient. Hung. Tomus 28 (1976), 1-4.

[12]. L. Bhatt and K.K. Dube, CR-submanifolds of trans-hyperbolic Sasakian manifold, ActaCienciaIndica 31 (2003), 91-96.

[13]. A. Bejancu,Geometry of CR-submanifolds, D. Reidel Publishing Company, Holland,1986.

[14]. D.E. Blair, Contact manifolds in Riemannian Geometry, Lecture Notes in Mathematics, Vol. 509, Springer-Verlag, Berlin, 1976. 